Article

\title{
Steroidal Saponins from the Rhizomes of Anemarrhena asphodeloides
}

\author{
Bing-You Yang, Jing Zhang, Yan Liu and Hai-Xue Kuang *
}

Key Laboratory of Chinese Materia Medica (Ministry of Education), Heilongjiang University of Chinese Medicine, Harbin 150040, China; ybywater@163.com (B.-Y.Y.); zhangjing823@yeah.net (J.Z.); lifeliuyan@163.com (Y.L.)

* Correspondence: hxkuang@yahoo.com; Tel.: +86-451-8219-3001

Academic Editors: Nancy D. Turner and Isabel C. F. R. Ferreira

Received: 19 May 2016; Accepted: 1 August 2016; Published: 17 August 2016

\begin{abstract}
Four new steroid saponins 1-4 were isolated from the rhizomes of Anemarrhena asphodeloides (Asparagaceae), as well as four known saponins: anemarsaponin B (5) timosaponin D (6), timosaponin E1 (7) anemarsaponin B II (8). Their structures were established through UV and NMR as well as MS data. All the compounds were evaluated for cytotoxicity against HepG2 and SGC7901 human cancer lines. Compounds $\mathbf{3}$ and $\mathbf{7}$ displayed medium antiproliferative activities on HepG2 and SGC7901 cells, with $\mathrm{IC}_{50}$ values of 43.90 and $57.90 \mu \mathrm{M}$, respectively.
\end{abstract}

Keywords: Asparagaceae; furostanol saponin; spirostanol saponin; MTT; cytotoxicity

\section{Introduction}

The rhizomes of Anemarrhena asphodeloides (Asparagaceae) have been used as traditional Chinese medicine for centuries. Phytochemical studies on Anemarrhena asphodeloides have led to the identification of a series of compounds, such as steroidal saponins, flavonoids, phenylpropanoids and alkaloids [1]. The most remarkable of the bioactive ingredients are the steroidal saponins. Recently, steroidal saponin studies were focused on a range of bioactivities, such as anti-inflammatory [2], antiplatelet $[3,4]$, and especially anti-tumor properties [5,6]. To explore its active components, we carried out a series of studies on the rhizomes of Anemarrhena asphodeloides. Our experiments have led to the isolations of four new steroidal saponins 1-4, which we have named anemarsaponin P-S, along with four known ones, including anemarsaponin B (5) [7], timosaponin D (6) [8], timosaponin E1 (7) [9], anemarsaponin B II (8) [10] (Figure 1).

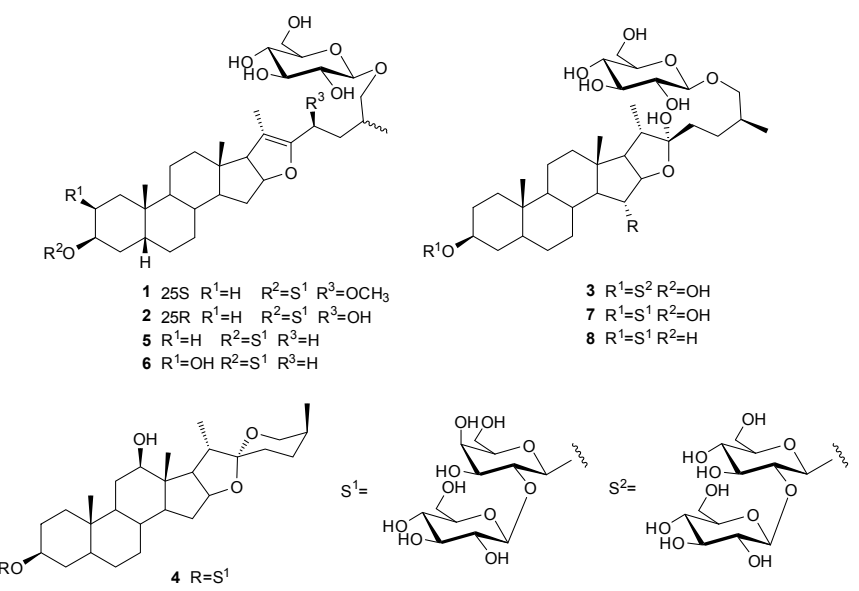

Figure 1. Structures of compounds 1-8 from Anemarrhena asphodeloides. 
The cytotoxicity of the isolated compounds has also been evaluated by the MTT method. This paper therefore reports the isolation, structural elucidation, and antiproliferative activities of steroidal saponins from Anemarrhena asphodeloides.

\section{Results}

\subsection{Structure Elucidation}

Anemarsaponin P (1) was obtained as an amorphous power and its molecular formula was $\mathrm{C}_{46} \mathrm{H}_{76} \mathrm{O}_{19}$ according to the HR-ESI-MS data $\left(m / z 955.4644[\mathrm{M}+\mathrm{Na}]^{+}\right)$(Figure 66 ). The corresponding ${ }^{1} \mathrm{H}-\mathrm{NMR}$ (Figure S7) data of the aglycone portion (Table 1) showed four methyl signals at $\delta_{\mathrm{H}} 0.66$ $(3 \mathrm{H}, \mathrm{s}), 0.99(3 \mathrm{H}, \mathrm{s}), 1.73(3 \mathrm{H}, \mathrm{s})$ and $1.12(3 \mathrm{H}, \mathrm{d}, J=6.8 \mathrm{~Hz})$, three anomeric protons at $\delta_{\mathrm{H}} 4.83(1 \mathrm{H}, \mathrm{d}$, $J=8.0 \mathrm{~Hz}), 5.28(1 \mathrm{H}, \mathrm{d}, J=7.6 \mathrm{~Hz})$ and $4.92(1 \mathrm{H}, \mathrm{d}, J=8.0 \mathrm{~Hz})$ and a methoxy group at $\delta_{\mathrm{H}} 3.32$ $(3 \mathrm{H}, \mathrm{s})$. The ${ }^{13} \mathrm{C}-\mathrm{NMR}$ spectrum (Figure $\mathrm{S} 8$ ) showed four methyl groups at $\delta_{C} 14.6,24.1,11.6$ and 17.9. Characteristic signals at 109.2 (C-20), 150.4 (C-22) and the secondary carbon signal at $\delta_{C} 75.5$ (C-26) indicated that compound 1 was a $\Delta^{20(22)}$-unsaturated furostanol saponin [11]. Comparison of the ${ }^{1} \mathrm{H}$ - and ${ }^{13} \mathrm{C}-\mathrm{NMR}$ spectra in 1 with those of anemarsaponin $\mathrm{B}(5)$ revealed the ring A-E portions and glycoside moiety of C-3 of the former were consistent with those of 5 . On the other hand, remarkable differences were indicated by the carbon signals from the ring F portion (C-22 C-27). The HMBC correlations between the methoxy signal $\left(\delta_{\mathrm{H}} 3.32\right)$ and $\mathrm{C}-23\left(\delta_{\mathrm{C}} 73.7\right)$ indicated that methoxy group should be placed in C-23, which was proved by the HMBC correlations from $\mathrm{H}-24_{\mathrm{a}}$ $\left(\delta_{\mathrm{H}} 2.07\right)$ to $\mathrm{C}-22\left(\delta_{\mathrm{C}} 150.4\right)$ (Figure 2a). The key NOESY correlations between H-23 and H-21/H-27 were indicative of $\alpha$-orientation for $\mathrm{H}-23$ (Figure $2 b$ ). Therefore, the methoxy group at $\mathrm{C}-23$ had a $\beta$-orientation. On the basis of above features, a $23 S$ configuration was established by reference to notation of $R, S$-configuration. The absolute configuration of $25 S$ in $\mathbf{1}$ was established by the chemical shift of $\mathrm{H}_{2}-26\left(\delta_{\mathrm{H}} 3.56\right.$ and $\left.4.15 \mathrm{ppm}, \Delta \delta=0.59\right)(\Delta \delta \geq 0.57 \mathrm{ppm})$ [11]. Thus, compound 1 was

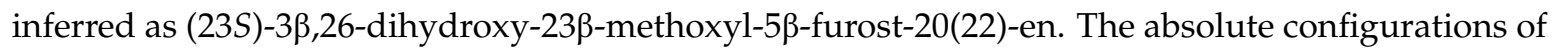
the $\beta$-glucose and $\beta$-galactose [12] were determined as D by GC analysis of their hydrolyzed forms. The linkage of the sugar in 1 was proved by long-range $\mathrm{HMBC}$ correlations between $\delta_{\mathrm{H}} 4.83\left(\mathrm{H}-1^{\prime}\right)$ and $\delta_{\mathrm{C}} 75.5\left(\mathrm{C}-26\right.$, aglycone), $\delta_{\mathrm{H}} 4.92\left(\mathrm{H}-1^{\prime \prime}\right)$ and $\delta_{\mathrm{C}} 75.3$ (C-3, aglycone) and $\delta_{\mathrm{H}} 5.28\left(\mathrm{H}-1^{\prime \prime \prime}\right)$ and $\delta_{\mathrm{C}}$ 81.8 (C-2" , 3-O- $\beta$-D-galactose) (Figure 2a, Table 2). Thus, the structure of compound 1 was deduced to

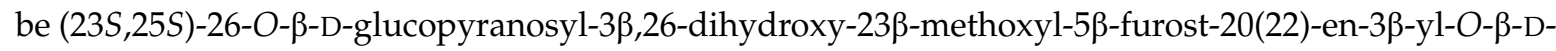
glucopyranosyl-(1 $\rightarrow 2)-\beta$-D-galactopyranoside.

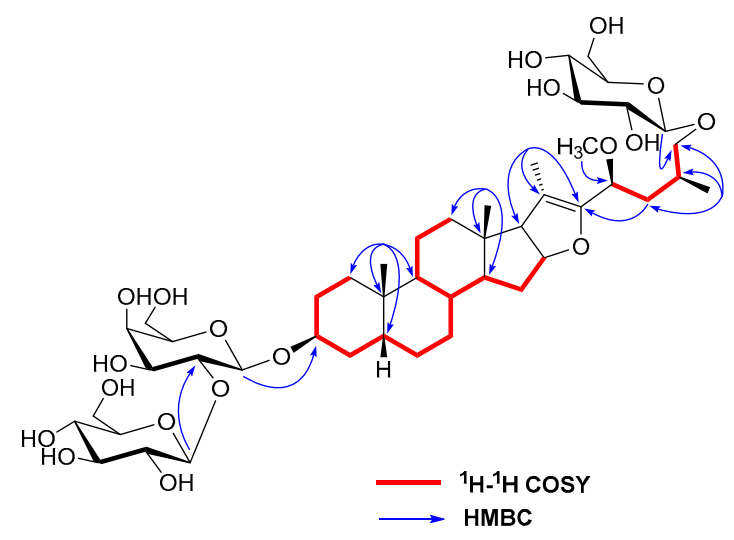

(a)

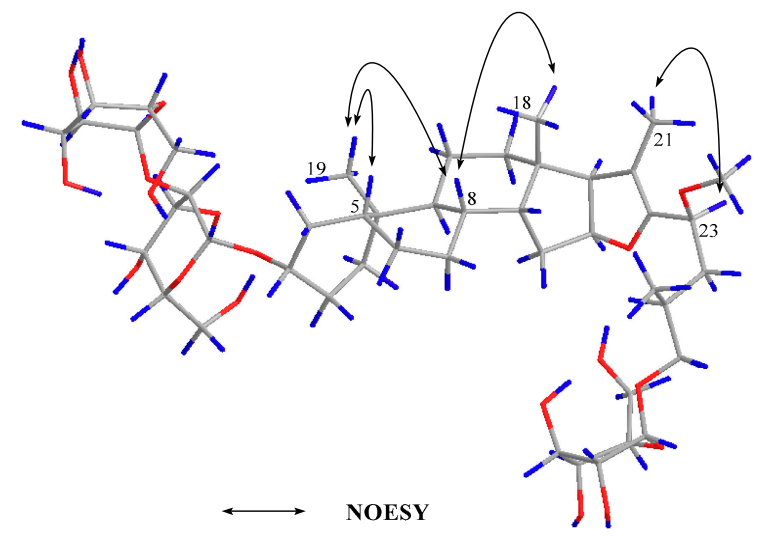

(b)

Figure 2. Key HMBC and ${ }^{1} \mathrm{H}_{-}{ }^{1} \mathrm{H}$ COSY correlations (a) of compound 1; Key NOESY correlations (b) of compound 1.

Anemarsaponin $Q$ (2) was an amorphous power and its molecular formula was $\mathrm{C}_{45} \mathrm{H}_{74} \mathrm{O}_{19}$ according to HR-ESI-MS at $m / z 941.4714[\mathrm{M}+\mathrm{Na}]^{+}$(Figure S9). The ${ }^{13} \mathrm{C}-\mathrm{NMR}$ spectrum data 
(Figure S11) (Table 1) showed four methyl groups at $\delta_{C} 14.6,24.0,11.7$ and 17.8. In addition, the carbon signals at $\delta_{C} 105.1(\mathrm{C}-20), 154.4$ (C-22) and the secondary C-26 carbon signal $\left(\delta_{C} 75.6\right)$ indicated that compound 2 was $\Delta^{20(22)}$-unsaturated furostanol saponin [11]. The ${ }^{13} \mathrm{C}-\mathrm{NMR}$ data of compound 2 was very similar to those of $\mathbf{1}$, except for the absence of a methoxy in $\mathbf{2}$. Significant differences were observed between 1 and 2 for C-22 C-27. The C-23 that appeared at $\delta_{C} 73.7$ of 1 was instead an upfield-shifted carbon at $\delta_{C} 63.9$ in compound 2 . The above data suggested that a hydroxy group existed at C-23 of 2, which was supported by the correlations from H-23 to H-24, H-24 to H-25, and H-25 to H-27 in the ${ }^{1} \mathrm{H}-{ }^{1} \mathrm{H}$ COSY spectrum of 2 (Figure S1).

Table 1. ${ }^{1} \mathrm{H}-\mathrm{NMR}$ and ${ }^{13} \mathrm{C}-\mathrm{NMR}$ data for aglycone moiety of compounds $\mathbf{1 - 4}$ (pyridine- $d_{5}$ ).

\begin{tabular}{|c|c|c|c|c|c|c|c|c|}
\hline \multirow{2}{*}{ No. } & \multicolumn{2}{|l|}{1} & \multicolumn{2}{|l|}{2} & \multicolumn{2}{|l|}{3} & \multicolumn{2}{|l|}{4} \\
\hline & $\delta_{\mathrm{H}}$ mult $(J, \mathrm{~Hz})$ & $\delta_{\mathrm{C}}$ & $\delta_{\mathrm{H}}$ mult $(J, \mathrm{~Hz})$ & $\delta_{\mathrm{C}}$ & $\delta_{\mathrm{H}}$ mult $(J, \mathrm{~Hz})$ & $\delta_{C}$ & $\delta_{\mathrm{H}}$ mult $(J, \mathrm{~Hz})$ & $\delta_{C}$ \\
\hline $1 \mathbf{a}$ & $1.49 \mathrm{~m}$ & 31.0 & $1.48 \mathrm{~m}$ & 31.0 & $1.48^{\mathrm{a}}$ & 31.6 & $1.47^{\mathrm{a}}$ & 31.0 \\
\hline $1 b$ & $1.87^{\mathrm{a}}$ & & $1.83^{\mathrm{a}}$ & & $1.88^{\mathrm{a}}$ & & $1.80^{\mathrm{a}}$ & \\
\hline $2 a$ & $1.21^{\mathrm{a}}$ & 27.0 & $1.23^{\mathrm{a}}$ & 27.0 & $1.45^{\mathrm{a}}$ & 27.3 & $1.51^{\mathrm{a}}$ & 26.7 \\
\hline $2 b$ & $1.84^{\mathrm{a}}$ & & $1.87^{\mathrm{a}}$ & & $1.92^{\mathrm{a}}$ & & $1.95 \mathrm{~m}$ & \\
\hline 3 & $4.28 \mathrm{~m}$ & 75.3 & $4.28 \mathrm{~m}$ & 75.3 & $4.30^{\mathrm{a}}$ & 75.7 & $4.31 \mathrm{~m}$ & 75.5 \\
\hline $4 a$ & $1.45^{\mathrm{a}}$ & 31.0 & $1.52 \mathrm{~m}$ & 31.0 & $1.80^{\mathrm{a}}$ & 31.2 & $1.81^{\mathrm{a}}$ & 31.0 \\
\hline $4 b$ & $1.81^{\mathrm{a}}$ & & $1.89^{\mathrm{a}}$ & & $1.82^{\mathrm{a}}$ & & $1.85^{\mathrm{a}}$ & \\
\hline 5 & $2.15 \mathrm{~m}$ & 37.0 & $2.18 \mathrm{~m}$ & 37.0 & $2.20 \mathrm{~m}$ & 37.4 & $2.16 \mathrm{~m}$ & 36.8 \\
\hline $6 a$ & $0.92 \mathrm{~m}$ & 26.8 & $0.98 \mathrm{~m}$ & 26.9 & $1.25^{\mathrm{a}}$ & 27.6 & $1.21 \mathrm{~m}$ & 27.1 \\
\hline $6 b$ & $1.82^{\mathrm{a}}$ & & $1.20^{\mathrm{a}}$ & & $1.87^{\mathrm{a}}$ & & $1.83^{\mathrm{a}}$ & \\
\hline $7 a$ & $1.51^{\mathrm{a}}$ & & $1.51^{\mathrm{a}}$ & & $1.50^{\mathrm{a}}$ & & $0.93 \mathrm{~m}$ & \\
\hline $7 \mathbf{b}$ & $1.98 \mathrm{~m}$ & 26.9 & $2.00 \mathrm{~m}$ & 26.9 & $2.31 \mathrm{~m}$ & 27.5 & $1.28^{\mathrm{a}}$ & 26.7 \\
\hline 8 & $1.38^{\mathrm{a}}$ & 35.1 & $1.40^{\mathrm{a}}$ & 35.2 & $1.86^{\mathrm{a}}$ & 36.9 & $1.54^{\mathrm{a}}$ & 34.7 \\
\hline 9 & $1.22^{\mathrm{a}}$ & 40.2 & $1.27^{\mathrm{a}}$ & 40.2 & $1.42^{\mathrm{a}}$ & 40.9 & $1.46^{\mathrm{a}}$ & 39.4 \\
\hline 10 & & 35.3 & & 35.3 & & 35.9 & & 35.3 \\
\hline $11 a$ & $1.15 \mathrm{~m}$ & 21.3 & $1.15^{\mathrm{a}}$ & 21.3 & $1.22^{\mathrm{a}}$ & 21.7 & $1.49^{\mathrm{a}}$ & 31.4 \\
\hline $11 b$ & $1.32 \mathrm{~m}$ & & $1.32 \mathrm{~m}$ & & $1.35 \mathrm{~m}$ & & $1.78 \mathrm{~m}$ & \\
\hline $12 a$ & $1.17^{\mathrm{a}}$ & & $1.15^{\mathrm{a}}$ & & $1.20^{\mathrm{a}}$ & & & \\
\hline $12 b$ & $1.70 \mathrm{~m}$ & 40.1 & $1.72 \mathrm{~d}(9.2)$ & 40.1 & $1.68 \mathrm{~m}$ & 41.6 & $3.53 \mathrm{dd}(4.0,10.0)$ & 79.5 \\
\hline 13 & & 44.0 & & 43.9 & & 41.8 & & 46.7 \\
\hline 14 & $0.81 \mathrm{~m}$ & 54.8 & $0.80 \mathrm{~m}$ & 54.8 & $1.53^{\mathrm{a}}$ & 61.4 & $1.10 \mathrm{~m}$ & 55.3 \\
\hline $15 a$ & $1.04^{\mathrm{a}}$ & 34.5 & $1.42^{\mathrm{a}}$ & 34.5 & $4.40^{\mathrm{a}}$ & 79.5 & $1.58 \mathrm{~m}$ & 31.9 \\
\hline $15 b$ & $2.05 \mathrm{~m}$ & & $2.07 \mathrm{dd}(5.6,12.4)$ & & & & $2.09 \mathrm{dd}(5.4,12.0)$ & \\
\hline 16 & $4.87 \mathrm{~m}$ & 84.8 & $4.85 \mathrm{~m}$ & 84.7 & $5.05 \mathrm{dd}(4,8.8)$ & 91.9 & $4.66 \mathrm{t}(5.4)$ & 81.4 \\
\hline 17 & $2.55 \mathrm{~d}(10.0)$ & 65.0 & $2.50 \mathrm{~d}(10.4)$ & 65.1 & $2.18^{\mathrm{a}}$ & 61.9 & $2.22^{\mathrm{a}}$ & 62.8 \\
\hline 18 & $0.66 \mathrm{~s}$ & 14.6 & $0.70 \mathrm{~s}$ & 14.6 & $0.95 \mathrm{~s}$ & 18.5 & $1.08 \mathrm{~s}$ & 11.2 \\
\hline 19 & $0.99 \mathrm{~s}$ & 24.1 & $0.99 \mathrm{~s}$ & 24.0 & $1.04 \mathrm{~s}$ & 24.7 & $1.00 \mathrm{~s}$ & 24.0 \\
\hline 20 & & 109.2 & & 105.1 & $2.26 \mathrm{~m}$ & 41.4 & $2.18 \mathrm{~m}$ & 43.6 \\
\hline 21 & $1.73 \mathrm{~s}$ & 11.6 & $1.75 \mathrm{~s}$ & 11.7 & $1.30 \mathrm{~d}(6.8)$ & 16.9 & $1.48 \mathrm{~d}(6.4)$ & 14.3 \\
\hline 22 & & 150.4 & & 154.4 & & 110.8 & & 110.0 \\
\hline 23 & $4.22 \mathrm{~m}$ & 73.7 & $4.92 \mathrm{~m}$ & 63.9 & $\begin{array}{c}2.09^{\mathrm{a}} \\
1.97 \mathrm{~m}\end{array}$ & 37.6 & $\begin{array}{c}2.18^{\mathrm{a}} \\
1.36 \mathrm{~m}\end{array}$ & 26.3 \\
\hline $24 a$ & $1.87^{\mathrm{a}}$ & 37.3 & 1.80 br.d (12.4) & 39.7 & $1.70 \mathrm{~m}$ & 28.9 & $1.30 \mathrm{~m}$ & 26.5 \\
\hline $24 b$ & $2.07^{\mathrm{a}}$ & & $2.40 \mathrm{~m}$ & & $2.07^{\mathrm{a}}$ & & $1.50^{\mathrm{a}}$ & \\
\hline 25 & $2.21 \mathrm{~m}$ & 31.1 & 2.48 br.d (10.4) & 31.0 & $1.92^{\mathrm{a}}$ & 34.9 & $1.62^{\mathrm{a}}$ & 27.6 \\
\hline $26 a$ & $3.56 \mathrm{~m}$ & 75.5 & 3.81 br.d (10.4) & 75.6 & $3.48 \mathrm{dd}(6.8,9.2)$ & 75.9 & 3.40 br.d (10.8) & 65.1 \\
\hline $26 b$ & $4.15^{\mathrm{a}}$ & & $4.07 \mathrm{~m}$ & & $4.08 \mathrm{~m}$ & & $4.13 \mathrm{~m}$ & \\
\hline 27 & $1.12 \mathrm{~d}(6.8)$ & 17.9 & $1.18 \mathrm{~d}(6.4)$ & 17.8 & $1.01 \mathrm{~d}(6.8)$ & 17.9 & $1.09 \mathrm{~d}(6.8)$ & 16.3 \\
\hline $\mathrm{OCH}_{3}$ & $3.32 \mathrm{~s}$ & 56.0 & & & & & & \\
\hline
\end{tabular}

a Overlapped signals.

The NOESY cross-peaks between H-23 and H-21 indicated the $\alpha$-orientation of $\mathrm{H}-23$ and the $\beta$-orientation of hydroxyl group for C-23 (Figure S2). Being similar to compound 1, the absolute configuration of $23 S$ was confirmed by reference to the notation of $R, S$-configuration. The $25 R$ configuration in 2 was proved by the protons of $\mathrm{H}_{2}-26\left(\delta_{\mathrm{H}} 3.81\right.$ and $\left.4.07 \mathrm{ppm}, \Delta \delta=0.26\right)$ $(\Delta \delta \leq 0.48 \mathrm{ppm})$ [11]. Compound 2 afforded D-glucose and D-galactose, identified by GC analysis of their acid hydrolysis derivatives. Compound 2 was thus established as $(23 S, 25 R)-26-$

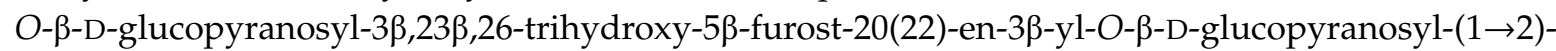
$\beta$-D-galactopyranoside. 
Anemarsaponin R (3) (Figure S12). had a molecular formula $\mathrm{C}_{45} \mathrm{H}_{76} \mathrm{O}_{20}$, based on HR-ESI-MS at $m / z 959.4840\left[\mathrm{M}+\mathrm{Na}^{+}\right.$. In the ${ }^{13} \mathrm{C}-\mathrm{NMR}$ spectrum (Figure S14) of 3 (Table 1) four methyl groups $\left(\delta_{C} 18.5,24.7,16.9\right.$ and 17.9$)$ and quaternary carbon $\left(\delta_{C} 110.8\right)$ suggested that compound 3 was a furostanol saponin [11]. Comparison of ${ }^{13} \mathrm{C}-\mathrm{NMR}$ data indicated the same skeleton in 3 and timosaponin E1 (7). The tiny differences between them were seen in the sugar moiety of C-3. Instead of glucose and galactose in 7, two glucoses were identified in 3 by the signals at $\delta_{C} 102.3,83.6,78.6,72.1,78.7,63.4$ and $\delta_{C} 106.4,77.5,78.4,72.3,79.0,63.2$ (Table 2). In addition, the existence of glucose was also confirmed by the coupling constant of $9.6 \mathrm{~Hz}$ in $\mathrm{H}-4$ for compound 3 instead of the typical one of galactose [13,14]. Meanwhile, the ${ }^{13} \mathrm{C}-\mathrm{NMR}$ data of the sugar residue matched those of anemarsaponin C [15]. Their sugar linkages were established by the existence of long-range HMBC correlations between $\delta_{\mathrm{H}} 4.81\left(\mathrm{H}-1^{\prime}\right)$ and $\delta_{\mathrm{C}} 75.9(\mathrm{C}-26$, aglycone), $\delta_{\mathrm{H}} 4.94\left(\mathrm{H}-1^{\prime \prime}\right)$ and $\delta_{\mathrm{C}} 75.7\left(\mathrm{C}-3\right.$, aglycone) and $\delta_{\mathrm{H}} 5.39\left(\mathrm{H}-1^{\prime \prime \prime}\right)$ and $\delta_{\mathrm{C}} 83.6\left(\mathrm{C}-2^{\prime \prime}\right.$, $3-O-\beta$-D-glucose) (Figure S3). The sugars of 3 were also only assignable to D-glucose by GC analysis of their chiral derivatives. The NOESY cross-peaks between the signals of $\mathrm{H}-23_{\mathrm{a}}$ and $\mathrm{H}-20, \mathrm{H}-15$ and $\mathrm{H}-18$ indicated the $\alpha$-orientations of the hydroxyl groups at C-15 and C-22 (Figure S4). In addition, the NOESY correlations between H-3 and H-1", H-5 and H-19 indicated the $\beta$-orientations of OH-3, H-5 (Figure S4). Thus, the structure of compound 3 was established as (25S)-26-O- $\beta$-D-glucopyranosyl-3 $\beta, 15 \alpha, 22 \alpha, 26$-tetrahydroxy- $5 \beta$-furost-3 $\beta$-yl-O- $\beta$-D-glucopyranosyl$(1 \rightarrow 2)-\beta$-D-glucopyranoside.

Table 2. ${ }^{1} \mathrm{H}-\mathrm{NMR}$ and ${ }^{13} \mathrm{C}-\mathrm{NMR}$ data for sugar portions of compounds $\mathbf{1}-\mathbf{4}$ (pyridine- $d_{5}$ ).

\begin{tabular}{|c|c|c|c|c|c|c|c|c|}
\hline \multirow{2}{*}{ No. } & \multicolumn{2}{|l|}{1} & \multicolumn{2}{|l|}{2} & \multicolumn{2}{|l|}{3} & \multicolumn{2}{|l|}{4} \\
\hline & $\delta_{\mathrm{H}}(\mathrm{mult}, J, \mathrm{~Hz})$ & $\delta_{\mathrm{C}}$ & $\delta_{\mathrm{H}}($ mult, $J, \mathrm{~Hz})$ & $\delta_{\mathrm{C}}$ & $\delta_{\mathrm{H}}(\mathrm{mult}, J, \mathrm{~Hz})$ & $\delta_{\mathrm{C}}$ & $\delta_{\mathrm{H}}($ mult, $J, \mathrm{~Hz})$ & $\delta_{\mathrm{C}}$ \\
\hline Glc-1 & $4.83 \mathrm{~d}(8.0)$ & 105.3 & $4.89 \mathrm{~d}(7.6)$ & 105.1 & $4.81 \mathrm{~d}(7.6)$ & 105.7 & & \\
\hline 2 & $4.01 \mathrm{~m}$ & 75.3 & $4.07^{\mathrm{a}}$ & 75.2 & $4.03^{\mathrm{a}}$ & 75.7 & & \\
\hline 3 & $3.84^{\mathrm{a}}$ & 78.5 & $3.97 \mathrm{~m}$ & 78.6 & 4.24 br.d (8.8) & 79.1 & & \\
\hline 4 & $4.20^{\mathrm{a}}$ & 71.7 & $4.22 \mathrm{t}(8.4)$ & 71.8 & 4.23 br.d (8.8) & 72.2 & & \\
\hline 5 & $4.21^{\mathrm{a}}$ & 78.6 & $4.24 \mathrm{~m}$ & 78.7 & $3.95^{\mathrm{a}}$ & 78.9 & & \\
\hline 6 & $\begin{array}{c}4.36 \mathrm{~m} \\
4.45 \mathrm{~d}(8.0)\end{array}$ & 62.9 & $\begin{array}{l}4.39 \mathrm{dd}(2.8,12.4) \\
4.55 \mathrm{dd}(5.6,12.4)\end{array}$ & 63.0 & $\begin{array}{c}4.32 \mathrm{dd}(3.2,14.8) \\
4.50 \text { br.d }(14.8)\end{array}$ & 63.3 & & \\
\hline Gal/Glc-1 & $4.92 \mathrm{~d}(8.0)$ & 102.6 & $4.93 \mathrm{~d}(7.6)$ & 102.6 & $4.94 \mathrm{~d}(7.6)$ & 102.3 & $4.93 \mathrm{~d}(8.0)$ & 102.6 \\
\hline 2 & $4.68 \mathrm{t}(7.6)$ & 81.8 & $4.69 \mathrm{t}(11.2)$ & 82.0 & $4.26 \mathrm{~m}$ & 83.6 & $4.70^{\mathrm{a}}$ & 81.9 \\
\hline 3 & $4.08 \mathrm{t}(7.6)$ & 77.0 & $4.10 \mathrm{~d}(11.2)$ & 76.9 & $4.32^{\mathrm{a}}$ & 78.6 & $4.27 \mathrm{dd}(3.2,9.6)$ & 75.3 \\
\hline 4 & $4.57 \mathrm{dd}(2.2,8.4)$ & 69.9 & $4.58^{\mathrm{a}}$ & 69.9 & 4.33 br.d (9.6) & 72.3 & $4.59 \mathrm{~d}(3.2)$ & 69.9 \\
\hline 5 & $4.03^{\mathrm{a}}$ & 76.7 & $4.04 \mathrm{~m}$ & 76.6 & $3.87 \mathrm{~m}$ & 78.7 & $4.03 \mathrm{t}(8.4)$ & 76.6 \\
\hline 6 & $\begin{array}{c}4.43 \text { br.d }(9.2) \\
4.39 \mathrm{dd}(3.6,9.2)\end{array}$ & 62.2 & $\begin{array}{c}4.40 \mathrm{dd}(1.8,12.0) \\
4.45 \text { br. d }(12.0)\end{array}$ & 62.2 & $\begin{array}{c}4.40 \mathrm{~d}(10.4) \\
4.54 \text { br.d }(10.4)\end{array}$ & 63.4 & $\begin{array}{l}4.40 \mathrm{dd}(1.2,8.4) \\
4.45 \mathrm{dd}(2.4,8.4)\end{array}$ & 62.2 \\
\hline Glc-1 & $5.28 \mathrm{~d}(7.6)$ & 106.1 & $5.30 \mathrm{~d}(8.0)$ & 106.2 & $5.39 \mathrm{~d}(8.0)$ & 106.4 & $5.30 \mathrm{~d}(7.6)$ & 106.2 \\
\hline 2 & $4.35 \mathrm{~m}$ & 75.6 & $4.31 \mathrm{t}(8.4)$ & 75.6 & $4.09^{\mathrm{a}}$ & 77.5 & $4.10 \mathrm{~m}$ & 77.0 \\
\hline 3 & $4.18^{\mathrm{a}}$ & 78.1 & $4.20 \mathrm{t}(8.4)$ & 78.1 & $4.28^{\mathrm{a}}$ & 78.4 & $4.22 \mathrm{t}(11.2)$ & 78.1 \\
\hline 4 & 4.30 br.d (9.6) & 71.8 & $4.32 \mathrm{~m}$ & 71.9 & 4.18 br.d (10.0) & 72.1 & 4.34 t (11.2) & 71.8 \\
\hline 5 & $3.84 \mathrm{~m}$ & 78.5 & $3.87 \mathrm{~m}$ & 78.4 & $4.26^{\mathrm{a}}$ & 79.0 & $3.86 \mathrm{~m}$ & 78.5 \\
\hline 6 & $\begin{array}{c}4.53 \mathrm{dd}(1.2,10.8) \\
4.47 \mathrm{~m}\end{array}$ & 62.9 & $\begin{array}{c}4.36 \text { dd }(4.0,9.6) \\
4.48 \text { br.d }(9.6)\end{array}$ & 62.9 & $\begin{array}{c}4.38 \mathrm{~d}(8.8) \\
4.57 \text { br.d (8.8) }\end{array}$ & 63.2 & $\begin{array}{l}4.47 \mathrm{dd}(3.6,8.4) \\
4.53 \mathrm{dd}(1.2,8.4)\end{array}$ & 62.8 \\
\hline
\end{tabular}

Anemarsaponin S (4) had a molecular formula $\mathrm{C}_{46} \mathrm{H}_{76} \mathrm{O}_{19}$, on the basis of HR-ESI-MS at $m / z 779.4193$ $[\mathrm{M}+\mathrm{Na}]^{+}$(Figure S15). In the ${ }^{1} \mathrm{H}-\mathrm{NMR}$ spectrum (Figure S16) of 4 (Table 1$)$ the signals at $\delta_{\mathrm{H}} 1.08(3 \mathrm{H}, \mathrm{s})$, $1.00(3 \mathrm{H}, \mathrm{s}), 1.48(3 \mathrm{H}, \mathrm{d}, J=6.4 \mathrm{~Hz})$ and $1.09(3 \mathrm{H}, \mathrm{d}, J=6.8 \mathrm{~Hz})$ were assignable to four methyl groups. Correspondingly, the signals at $\delta_{C} 11.2,24.0,14.3$ and 16.3 also demonstrated the existences of four methyl groups. In addition, the signal at $\delta_{C} 110.0$ was assignable to the characteristic $C-22$ carbon signal of a spirostanol saponin. Comparison of the carbon signals revealed multiple similarities between 4 and (25R)-5 $\beta$-spirostane-3 $\beta, 12 \beta$-diol-3-O- $\beta$-D-glucopyranosyl-( $1 \rightarrow 2)-\beta$-D-galacto-pyranoside [16], excluding the $\mathrm{F}$ ring. In the comparison of the $\mathrm{F}$ ring signals $\left(\delta_{\mathrm{C}} 32.2,29.6,31.0,67.2,17.7\right)$, apparent differences were observed at $\delta_{C} 26.3,26.5,27.6,65.1,16.3$ of 4 . Meanwhile, the ${ }^{13} \mathrm{C}-\mathrm{NMR}$ data of $\mathrm{F}$ ring in 1 were consistent with markogenin 3-O-D-glucopyranosyl-(1 $\rightarrow 2)-\beta$-D-galactopyranoside [17]. Certainly, 
both the shift of $\mathrm{H}_{3}-27\left(\delta_{\mathrm{H}} 1.09, \mathrm{~d}, J=6.8 \mathrm{~Hz}\right)$ and $\mathrm{H}_{2}-26$ proton chemistry shift $\left(\delta_{\mathrm{H}} 3.40\right.$ and 4.13) directly confirmed a $25 S$ configuration in 4 [18]. Therefore, compound 4 was formulated as (25S)-3 $\beta, 12 \beta$-dihydroxy-5 $\beta$-spirostane-3 $\beta$-yl-O- $\beta$-D-glucopyranosyl-( $1 \rightarrow 2)-\beta$-D-galactopyranoside.

By comparison of NMR data with those reported, the four known compounds were established as anemarsaponin B (5) [7], timosaponin D (6) [8], timosaponin E1 (7) [9], anemarsaponin B II (8) [10].

\subsection{Cytotoxic Activity}

Anemarsaponins P-S (compounds 1-4), anemarsaponin B (5), timosaponin D (6), timosaponin E1 (7) and anemarsaponin B II (8) were evaluated for their in vitro cytotoxic activities against two human tumor cell lines (HepG2 and SGC7901) through the MTT method. Among them, anemarsaponin R (3) showed medium cytotoxicity against HepG2 cells, with an $\mathrm{IC}_{50}$ value of $43.90 \mu \mathrm{M}$. Timosaponin E1 (7) exhibited medium cytotoxicity against SGC7901 cells, with an $\mathrm{IC}_{50}$ value of $57.90 \mu \mathrm{M}$. The other compounds did not show significant cytotoxicity $\left(\mathrm{IC}_{50}>100 \mu \mathrm{M}\right)$ (Table 3). The dose dependent of cell viabilityon HepG2 (a) and SGC7901 (b) for compounds (Figure 3).

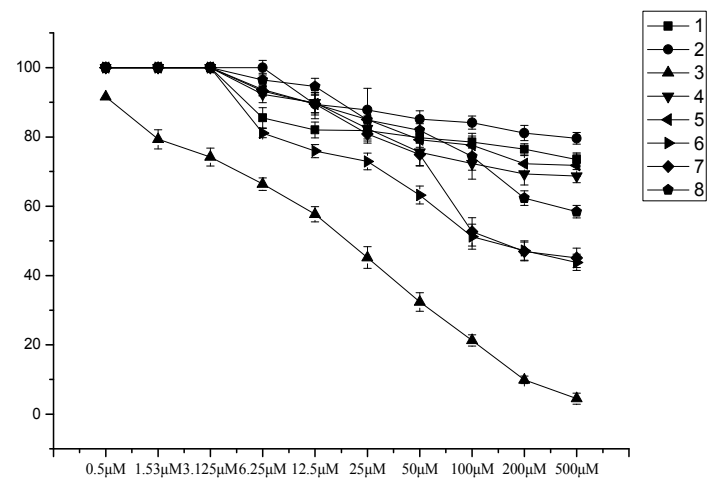

(a)

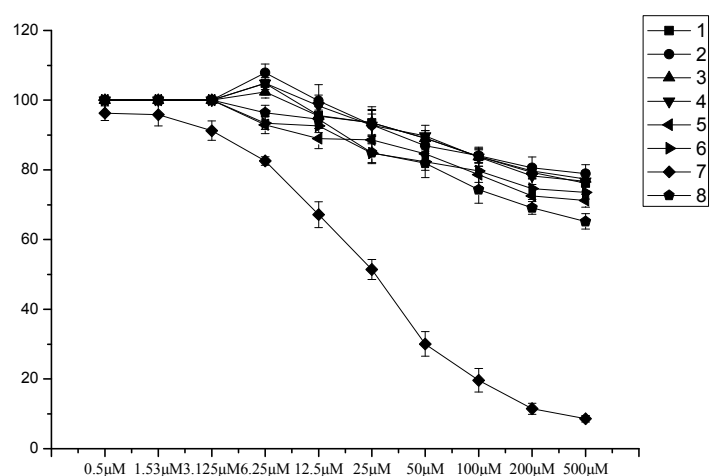

(b)

Figure 3. (a) Inhibition of HepG2 cell proliferation by the tested compounds; (b) Inhibition of SGC7901 cell proliferation by the tested compounds.

Table 3. Cytotoxicities of compounds 1-8.

\begin{tabular}{cccccc}
\hline \multirow{2}{*}{ Compounds } & \multicolumn{2}{c}{ IC $_{\mathbf{5 0}}(\boldsymbol{\mu M})$} & \multirow{2}{*}{ Compounds } & \multicolumn{2}{c}{ IC $_{\mathbf{5 0}}(\boldsymbol{\mu M})$} \\
\cline { 2 - 3 } \cline { 5 - 6 } & HepG2 & SGC7901 & & HepG2 & SGC7901 \\
\hline $\mathbf{1}$ & $>100$ & $>100$ & $\mathbf{6}$ & $>100$ & $>100$ \\
$\mathbf{2}$ & $>100$ & $>100$ & $\mathbf{7}$ & $>100$ & $57.90 \pm 2.88$ \\
$\mathbf{3}$ & $43.90 \pm 3.36$ & $>100$ & $\mathbf{8}$ & $>100$ & $>100$ \\
$\mathbf{4}$ & $>100$ & $>100$ & doxorubicin & $8.20 \pm 1.25$ & $6.25 \pm 2.18$ \\
$\mathbf{5}$ & $>100$ & $>100$ & & & \\
\hline
\end{tabular}

\section{Experimental Section}

\subsection{General Experimental Procedures}

Optical rotations were obtained on a P-2000 polarimeter (JASCO, Tokyo, Japan). IR spectra were obtained on a FTIR-8400S instrument (Shimadzu, Kyoto, Japan). UV spectra were obtained on a Shimadzu UV-1601 instrument. NMR spectra were obtained on DPX 400 NMR instrument (Bruker, Rheinstetten, Germany). HR-ESI-MS were recorded on a Xevo-TOF-MS ${ }^{\mathrm{TM}}$ instrument (Waters, Milford, MA, USA). HPLC was performed by using a Waters 515 HPLC system coupled with a Waters 2414 refractive index detector. A Waters XBridge preparative $C_{18}$ column $(19 \times 250 \mathrm{~mm}$, $10 \mu \mathrm{m}$ ) was used. Macroporous absorption resin D-101 (Cangzhou Bon Adsorber Technology Co., Ltd., Cangzhou, China), MPLC (C-610, Büchi, Flawil Switzerland) and ODS silica gel (YMC Ltd., 
Kyoto, Japan) were used for column chromatography. $\mathrm{MeOH}$ and EtOH were analytical grade and purchased from Tian-Jin Fu Yu Co., Ltd. (Tianjin, China). MeCN was HPLC grade and obtained from J\&K Scientific Ltd. (Beijing, China).

\subsection{Plant Material}

The rhizomes of Anemarrhena asphodeloides Bunge were collected at Bozhou Country, Anhui Province, People's Republic of China, in September 2014, and identified by Ruifeng Fan from Heilongjiang University of Chinese Medicine. A voucher specimen (20140930) has been deposited in the laboratory.

\subsection{Extration and Isolation}

The dried slice of rhizomes from Anemarrhena asphodeloides $(56 \mathrm{~kg})$ were extracted three times with hot water $(280 \mathrm{~L})$ under reflux. The solutions were combined and evaporated to give a residue $\left(22.09 \mathrm{~kg}\right.$ ). The crude was suspended with EtOAc, $n$ - $\mathrm{BuOH}, \mathrm{H}_{2} \mathrm{O}$, respectively. The $n$ - $\mathrm{BuOH}$ elute was concentrated under vacuum to yield the $n-\mathrm{BuOH}$-soluble fraction.

A part of the fraction $(120 \mathrm{~g})$ was chromatographed by MPLC, eluted with a stepwise gradient of $\mathrm{MeOH}-\mathrm{H}_{2} \mathrm{O}$ and finally with $\mathrm{MeOH}$, giving 20 subfractions. Fr.5 (3.1 g) was subjected to ODS column chromatography, eluted with a gradient of $\mathrm{MeOH}-\mathrm{H}_{2} \mathrm{O}$ (4:6 to 1:0) to yield Fr.5-1 Fr.5-4. Fr.5-4 (0.8 g) was separated by preparative HPLC $\left(5.0 \mathrm{~mL} / \mathrm{min}, 15 \% \mathrm{MeCN}^{-} \mathrm{H}_{2} \mathrm{O}\right)$ and yielded compound $4(4 \mathrm{mg})$. Fr.17 $(0.2 \mathrm{~g})$ was purified with HPLC $\left(5.0 \mathrm{~mL} / \mathrm{min}, 27 \% \mathrm{MeCN}^{-} \mathrm{H}_{2} \mathrm{O}\right)$ to give compound $7(6 \mathrm{mg})$. Fr.18 (25 g) was chromatographed over an ODS column to afford Fr.18-1 Fr.18-5. Compound 5 (2.9 g)

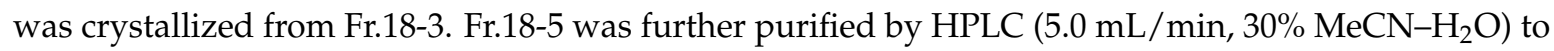
afford compounds $\mathbf{1}(5 \mathrm{mg}), \mathbf{2}(28 \mathrm{mg})$ and $\mathbf{3}(8 \mathrm{mg})$. Fr.19 $(2 \mathrm{~g})$ was purified by HPLC $(5.0 \mathrm{~mL} / \mathrm{min}$, $\left.38 \% \mathrm{MeCN}-\mathrm{H}_{2} \mathrm{O}\right)$ to give compounds $6(7 \mathrm{mg})$ and $8(57 \mathrm{mg})$.

Anemarsaponin P (1). White amorphous power; $[\alpha]_{\mathrm{D}}^{21.7}-67$ (c 2.0, $\left.\mathrm{MeOH}\right)$; UV (MeOH) $\gamma_{\max } 217 \mathrm{~nm}$; $\mathrm{CD} \Delta \varepsilon+1.5(231 \mathrm{~nm})$; IR (KBr) $v_{\max } 3454,2930,1646,1405,1070 \mathrm{~cm}^{-1} ;{ }^{1} \mathrm{H}-$ and ${ }^{13} \mathrm{C}-\mathrm{NMR}$ data (Figures $\mathrm{S} 7$ and S8), see Tables 1 and 2; HR-ESI-MS $m / z$ 955.4644 [M + Na] ${ }^{+}$(calc. for $\mathrm{C}_{46} \mathrm{H}_{76} \mathrm{O}_{19}$ 955.4878).

Anemarsaponin Q (2). White amorphous power; $[\alpha]_{\mathrm{D}}^{23.2}-7.1(c 1.7, \mathrm{MeOH}) ; \mathrm{UV}(\mathrm{MeOH}) \gamma_{\max } 211 \mathrm{~nm}$; $\mathrm{CD} \Delta \varepsilon+1.7(232 \mathrm{~nm})$; IR (KBr) $v_{\max } 2418,2931,1067,1033 \mathrm{~cm}^{-1} ;{ }^{1} \mathrm{H}$ - and ${ }^{13} \mathrm{C}-\mathrm{NMR}$ data (Figures S10 and S11), see Tables 1 and 2; HR-ESI-MS $m / z 941.4714$ [M + Na] (calc. for $\mathrm{C}_{45} \mathrm{H}_{74} \mathrm{O}_{19}$ 941.4722).

Anemarsaponin R (3). White amorphous power; $[\alpha]_{\mathrm{D}}^{21.8}-38.0$ (c 2.0, MeOH); IR (KBr) $v_{\max } 3465,2935$, 1397, $1080 \mathrm{~cm}^{-1} ;{ }^{1} \mathrm{Hp}$ and ${ }^{13} \mathrm{C}-\mathrm{NMR}$ data (Figures $\mathrm{S} 13$ and S14), see Tables 1 and 2; HR-ESI-MS $\mathrm{m} / z$ $959.4840[\mathrm{M}+\mathrm{Na}]^{+}$(calc. for $\mathrm{C}_{45} \mathrm{H}_{76} \mathrm{O}_{20}$ 959.4828).

Anemarsaponin S (4). White amorphous power; $[\alpha]_{\mathrm{D}}^{22}-5.0$ (c 2.0, MeOH); IR (KBr) $v_{\max } 3428,2930$, $1397,1070 \mathrm{~cm}^{-1} ;{ }^{1} \mathrm{H}$ - and ${ }^{13} \mathrm{C}-\mathrm{NMR}$ data (Figures $\mathrm{S} 16$ and S17), see Tables 1 and 2; HR-ESI-MS $\mathrm{m} / \mathrm{z}$ $779.4193[\mathrm{M}+\mathrm{H}]^{+}$(calc. for $\left.\mathrm{C}_{39} \mathrm{H}_{64} \mathrm{O}_{14} 779.4194\right)$.

Anemarsaponin B (5). White amorphous power; ${ }^{1} \mathrm{H}-\mathrm{NMR}(400 \mathrm{MHz}$, Pyridine) $\delta: 0.70(3 \mathrm{H}, \mathrm{s}, \mathrm{H}-18)$, $1.00(3 \mathrm{H}, \mathrm{s}, \mathrm{H}-19), 1.64(3 \mathrm{H}, \mathrm{s}, \mathrm{H}-21), 1.04(3 \mathrm{H}, \mathrm{d}, J=6.4 \mathrm{~Hz}, \mathrm{H}-27), 4.95\left(1 \mathrm{H}, \mathrm{d}, J=8.0 \mathrm{~Hz}, \mathrm{H}-1^{\prime \prime}\right)$, $4.85\left(1 \mathrm{H}, \mathrm{d}, J=7.6 \mathrm{~Hz}, \mathrm{H}-1^{\prime \prime \prime}\right), 5.31\left(1 \mathrm{H}, \mathrm{d}, J=7.6 \mathrm{~Hz}, \mathrm{H}-1^{\prime}\right) ;{ }^{13} \mathrm{C}-\mathrm{NMR}(100 \mathrm{MHz}$, Pyridine) $\delta: 31.0$ (C-1), 27.0 (C-2), 75.3 (C-3), 31.0 (C-4), 37.0 (C-5), 26.9 (C-6), 26.9 (C-7), 35.2 (C-8), 40.2 (C-9), 35.3 (C-10), 21.3 (C-11), 40.1 (C-12), 43.9 (C-13), 54.8 (C-14), 31.4 (C-15), 84.6 (C-16), 64.7 (C-17), 14.4 (C-18), 24.0 (C-19), 103.6 (C-20), 11.9 (C-21), 152.4 (C-22), 34.5 (C-23), 23.7 (C-24), 33.7 (C-25), 75.3 (C-26), $17.2(\mathrm{C}-27), 102.6\left(\mathrm{C}-1^{\prime \prime}\right), 82.0\left(\mathrm{C}-2^{\prime \prime}\right), 77.0\left(\mathrm{C}-3^{\prime \prime}\right), 69.9\left(\mathrm{C}-4^{\prime \prime}\right), 76.7\left(\mathrm{C}-5^{\prime \prime}\right), 62.2\left(\mathrm{C}-6^{\prime \prime}\right), 106.2\left(\mathrm{C}-1^{\prime \prime \prime}\right)$, $75.6\left(\mathrm{C}-2^{\prime \prime \prime}\right), 78.1\left(\mathrm{C}-3^{\prime \prime \prime}\right), 71.8\left(\mathrm{C}-4^{\prime \prime \prime}\right), 78.5\left(\mathrm{C}-5^{\prime \prime \prime}\right), 62.8\left(\mathrm{C}-6^{\prime \prime \prime}\right), 105.2\left(\mathrm{C}-1^{\prime}\right), 75.3\left(\mathrm{C}-2^{\prime}\right), 78.6\left(\mathrm{C}-3^{\prime}\right)$, $71.8\left(\mathrm{C}-4^{\prime}\right), 78.7\left(\mathrm{C}-5^{\prime}\right), 62.9\left(\mathrm{C}-6^{\prime}\right)$. 
Timosaponin D (6). White amorphous power; ${ }^{1} \mathrm{H}-\mathrm{NMR}(400 \mathrm{MHz}$, Pyridine) $\delta: 0.70(3 \mathrm{H}, \mathrm{s}, \mathrm{H}-18)$, $1.00(3 \mathrm{H}, \mathrm{s}, \mathrm{H}-19), 1.64(3 \mathrm{H}, \mathrm{s}, \mathrm{H}-21), 1.05(3 \mathrm{H}, \mathrm{d}, J=6.4 \mathrm{~Hz}, \mathrm{H}-27), 4.99\left(1 \mathrm{H}, \mathrm{d}, J=7.6 \mathrm{~Hz}, \mathrm{H}-1^{\prime \prime}\right), 4.82(1 \mathrm{H}$, $\left.\mathrm{d}, J=7.6 \mathrm{~Hz}, \mathrm{H}-1^{\prime \prime \prime}\right), 5.28\left(1 \mathrm{H}, \mathrm{d}, J=7.6 \mathrm{~Hz}, \mathrm{H}-1^{\prime}\right) ;{ }^{13} \mathrm{C}-\mathrm{NMR}$ (100 MHz, Pyridine) $\delta: 40.1(\mathrm{C}-1), 67.2(\mathrm{C}-2)$, 82.0 (C-3), 31.9 (C-4), 36.6 (C-5), 26.3 (C-6), 26.9 (C-7), 35.3 (C-8), 41.4 (C-9), 37.1 (C-10), 21.5 (C-11), 40.6 (C-12), 43.8 (C-13), 54.7 (C-14), 31.4 (C-15), 84.6 (C-16), 64.7 (C-17), 14.4 (C-18), 23.9 (C-19), 103.4 (C-20), 11.8 (C-21), 152.4 (C-22), 34.4 (C-23), 23.7 (C-24), 33.7 (C-25), 75.3 (C-26), 17.2 (C-27), $103.6\left(\mathrm{C}-1^{\prime \prime}\right), 81.8\left(\mathrm{C}-2^{\prime \prime}\right), 75.3\left(\mathrm{C}-3^{\prime \prime}\right), 69.8\left(\mathrm{C}-4^{\prime \prime}\right), 77.0\left(\mathrm{C}-5^{\prime \prime}\right), 62.1\left(\mathrm{C}-6^{\prime \prime}\right), 106.2\left(\mathrm{C}-1^{\prime \prime \prime}\right), 77.0\left(\mathrm{C}-2^{\prime \prime \prime}\right)$, $78.1\left(\mathrm{C}-3^{\prime \prime \prime}\right), 71.8\left(\mathrm{C}-4^{\prime \prime \prime}\right), 78.6\left(\mathrm{C}-5^{\prime \prime \prime}\right), 62.9\left(\mathrm{C}-6^{\prime \prime \prime}\right), 105.2\left(\mathrm{C}-1^{\prime}\right), 75.3\left(\mathrm{C}-2^{\prime}\right), 78.6\left(\mathrm{C}-3^{\prime}\right), 71.8\left(\mathrm{C}-4^{\prime}\right)$, $78.6\left(\mathrm{C}-5^{\prime}\right), 62.9\left(\mathrm{C}-6^{\prime}\right)$.

Timosaponin E1 (7). White amorphous power; ${ }^{1} \mathrm{H}-\mathrm{NMR}(400 \mathrm{MHz}$, Pyridine) $\delta: 0.81(3 \mathrm{H}, s, \mathrm{H}-18)$, $1.00(3 \mathrm{H}, \mathrm{s}, \mathrm{H}-19), 1.28(3 \mathrm{H}, \mathrm{d}, J=6.8 \mathrm{~Hz}, \mathrm{H}-21), 1.01(3 \mathrm{H}, \mathrm{d}, J=6.8 \mathrm{~Hz}, \mathrm{H}-27), 4.81(1 \mathrm{H}, \mathrm{d}, J=7.6 \mathrm{~Hz}$, H-1' $), 4.96\left(1 \mathrm{H}, \mathrm{d}, J=7.6 \mathrm{~Hz}, \mathrm{H}-1^{\prime \prime \prime}\right), 5.26\left(1 \mathrm{H}, \mathrm{d}, J=7.6 \mathrm{~Hz}, \mathrm{H}-1^{\prime}\right) ;{ }^{13} \mathrm{C}-\mathrm{NMR}$ (100 MHz, Pyridine)

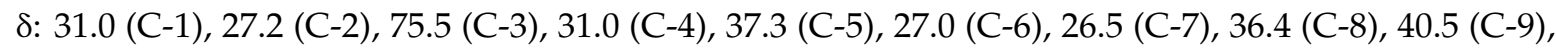
35.4 (C-10), 21.4 (C-11), 41.3 (C-12), 41.6 (C-13), 60.8 (C-14), 79.1 (C-15), 91.4 (C-16), 61.4 (C-17), 18.0 (C-18), 24.1 (C-19), 40.9 (C-20), 16.5 (C-21), 110.4 (C-22), 37.1 (C-23), 28.4 (C-24), 34.5 (C-25), 75.5 (C-26), 17.5 (C-27), $103.3\left(\mathrm{C}-1^{\prime \prime}\right), 81.8\left(\mathrm{C}-2^{\prime \prime}\right), 75.3\left(\mathrm{C}-3^{\prime \prime}\right), 69.9\left(\mathrm{C}-4^{\prime \prime}\right), 76.8\left(\mathrm{C}-5^{\prime \prime}\right), 62.1\left(\mathrm{C}-6^{\prime \prime}\right)$, $106.2\left(\mathrm{C}-1^{\prime \prime \prime}\right), 77.0\left(\mathrm{C}-2^{\prime \prime \prime}\right), 78.1\left(\mathrm{C}-3^{\prime \prime \prime}\right), 71.7\left(\mathrm{C}-4^{\prime \prime \prime}\right), 78.4\left(\mathrm{C}-5^{\prime \prime \prime}\right), 62.9\left(\mathrm{C}-6^{\prime \prime \prime}\right), 105.2\left(\mathrm{C}-1^{\prime}\right), 75.3\left(\mathrm{C}-2^{\prime}\right)$, $78.7\left(\mathrm{C}-3^{\prime}\right), 71.8\left(\mathrm{C}-4^{\prime}\right), 78.5\left(\mathrm{C}-5^{\prime}\right), 62.9\left(\mathrm{C}-6^{\prime}\right)$.

Anemarsaponin BII (8). White amorphous power; ${ }^{1} \mathrm{H}-\mathrm{NMR}(400 \mathrm{MHz}$, Pyridine) $\delta: 0.81$ (3H, s, H-18), $0.99(3 \mathrm{H}, \mathrm{s}, \mathrm{H}-19), 1.32(3 \mathrm{H}, \mathrm{d}, J=6.8 \mathrm{~Hz}, \mathrm{H}-21), 1.02(3 \mathrm{H}, \mathrm{d}, J=6.8 \mathrm{~Hz}, \mathrm{H}-27), 4.82(1 \mathrm{H}, \mathrm{d}, J=8.0 \mathrm{~Hz}$, H-1" $), 4.93\left(1 \mathrm{H}, \mathrm{d}, J=7.6 \mathrm{~Hz}, \mathrm{H}-1^{\prime \prime \prime}\right), 5.30\left(1 \mathrm{H}, \mathrm{d}, J=7.6 \mathrm{~Hz}, \mathrm{H}-1^{\prime}\right) ;{ }^{13} \mathrm{C}-\mathrm{NMR}$ (100 MHz, Pyridine) 8: 31.4 (C-1), 27.5 (C-2), 75.7 (C-3), 31.4 (C-4), 37.4 (C-5), 27.5 (C-6), 27.3 (C-7), 36.0 (C-8), 40.7 (C-9), 35.7 (C-10), 21.6 (C-11), 40.9 (C-12), 41.7 (C-13), 56.9 (C-14), 32.9 (C-15), 81.7 (C-16), 64.5 (C-17), 17.2 (C-18), 24.5 (C-19), 41.1 (C-20), 17.0 (C-21), 111.1 (C-22), 37.6 (C-23), 28.8 (C-24), 34.9 (C-25), 75.9 (C-26), 17.9 (C-27), $103.0\left(\mathrm{C}-1^{\prime \prime}\right), 82.3\left(\mathrm{C}-2^{\prime \prime}\right), 77.4\left(\mathrm{C}-3^{\prime \prime}\right), 70.3\left(\mathrm{C}-4^{\prime \prime}\right), 77.1\left(\mathrm{C}-5^{\prime \prime}\right), 63.2\left(\mathrm{C}-6^{\prime \prime}\right)$, $106.6\left(\mathrm{C}-1^{\prime \prime \prime}\right), 76.0\left(\mathrm{C}-2^{\prime \prime \prime}\right), 78.5\left(\mathrm{C}-3^{\prime \prime \prime}\right), 72.2\left(\mathrm{C}-4^{\prime \prime \prime}\right), 78.9\left(\mathrm{C}-5^{\prime \prime \prime}\right), 63.2\left(\mathrm{C}-6^{\prime \prime \prime}\right), 105.6\left(\mathrm{C}-1^{\prime}\right), 75.7\left(\mathrm{C}-2^{\prime}\right)$, $79.1\left(\mathrm{C}-3^{\prime}\right), 72.2\left(\mathrm{C}-4^{\prime}\right), 79.0\left(\mathrm{C}-5^{\prime}\right), 62.6\left(\mathrm{C}-6^{\prime}\right)$.

\subsection{Acid Hydrolysis and GC Analysis}

The hydrolysis and GC analysis of the four new compounds were carried out for the chiral sugar derivatives. Compounds 1-4 (2 mg) were heated with $5 \mathrm{~mL} 2 \mathrm{M} \mathrm{HCl}$ at $90^{\circ} \mathrm{C}$ for $3 \mathrm{~h}$. The mixtures were extracted with EtOAc ( $5 \mathrm{~mL}$ ) for three times. The sugar residue was dispersed with $1 \mathrm{~mL}$ pyridine and reacted with L-cysteine methyl ester hydrochloride $(1.5 \mathrm{mg})$ at $60^{\circ} \mathrm{C}$ for $1 \mathrm{~h}$. Then $150 \mu \mathrm{L}$ of HMDS-TMCS (hexamethyldisilazane-trimethylchlorosilane, 3:1) was added into the mixture that was further reacted at $60^{\circ} \mathrm{C}$ for $30 \mathrm{~min}$. The supernatant of the mixture was evaporated to dryness with a $\mathrm{N}_{2}$ stream. The residue was separated with $\mathrm{H}_{2} \mathrm{O}(0.1 \mathrm{~mL})$ and $n$-hexane $(0.1 \mathrm{~mL})$, and the supernatant layer $(1 \mu \mathrm{L})$ was analyzed by GC. The configurations of the sugar portion for compounds 1-4 were determined by comparison the retention times of their derivatives with those of standard D-glucose $\left(t_{R}=15.68 \mathrm{~min}\right)$ and D-galactose $\left(t_{R}=13.47 \mathrm{~min}\right)$ [19].

\subsection{Cytotoxic Activity}

The isolated compounds were evaluated for their in vitro antiproliferative activities by the MTT method. Doxorubicin was used as positive control (Table 3). Two cell lines, HepG2 and SGC7901, were obtained from the Shanghai Institute of Biochemistry and Cell Biology (Shanghai, China). They were cultured in RPMI 1640 supplemented with 10\% FBS, $100 \mathrm{IU} / \mathrm{mL}$ penicillin, $100 \mu \mathrm{g} / \mathrm{mL}$ streptomycin in $5 \% \mathrm{CO}_{2}$ at $37{ }^{\circ} \mathrm{C}$. Cells were cultured in 96-well plates for $24 \mathrm{~h}$ with $100 \mu \mathrm{L}$ complete medium, followed by treating with compounds at different concentrations. $20 \mu \mathrm{L}$ MTT $(5 \mathrm{mg} / \mathrm{mL}$ in PBS) was added in the 96-well plates for another $4 \mathrm{~h}$. The solutions were assayed at $490 \mathrm{~nm}$ 
using a VICTOR-X3 ELISA instrument (PerkinElmer, Waltham, MA, USA), after the precipitates were dissolved in DMSO [20]. The cytotoxicities of compounds against HepG2 and SGC7901 were calculated and expressed as $\mathrm{IC}_{50}$ values.

\section{Conclusions}

As described in the introduction, in recent years steroidal saponins have become a research hotspot due to their multiple and strong bioactivities, especially their cytotoxic activities against a series of tumor cell lines $[5,6,21]$. In this paper, four new saponins were isolated from Anemarrhena asphodeloides their strictures elucidated, and their antiproliferative activities against HepG2 and SGC7901 were evaluated. Obvious differences were observed between the antiproliferative activities of compounds 3 , 7 and the others. The above results represent a contribution to the discovery of new active ingredients and lead compounds and provide an experimental and scientific basis for drug design and drug discovery.

Supplementary Materials: Supplementary materials can be accessed at: http://www.mdpi.com/1420-3049/21/ 8/1075/s1.

Acknowledgments: This project was supported by Major State Basic Research Development Program (973 Program)of China (2013CB531800) and National Natural Science Foundation of China (No. 81274103).

Author Contributions: B.-Y.Y. and H.-X.K. designed the experiments; J.Z. performed the experiments; J.Z. wrote the paper. Y.L. modified the paper; All authors read and approved the manuscript.

Conflicts of Interest: The authors declare no conflict of interest.

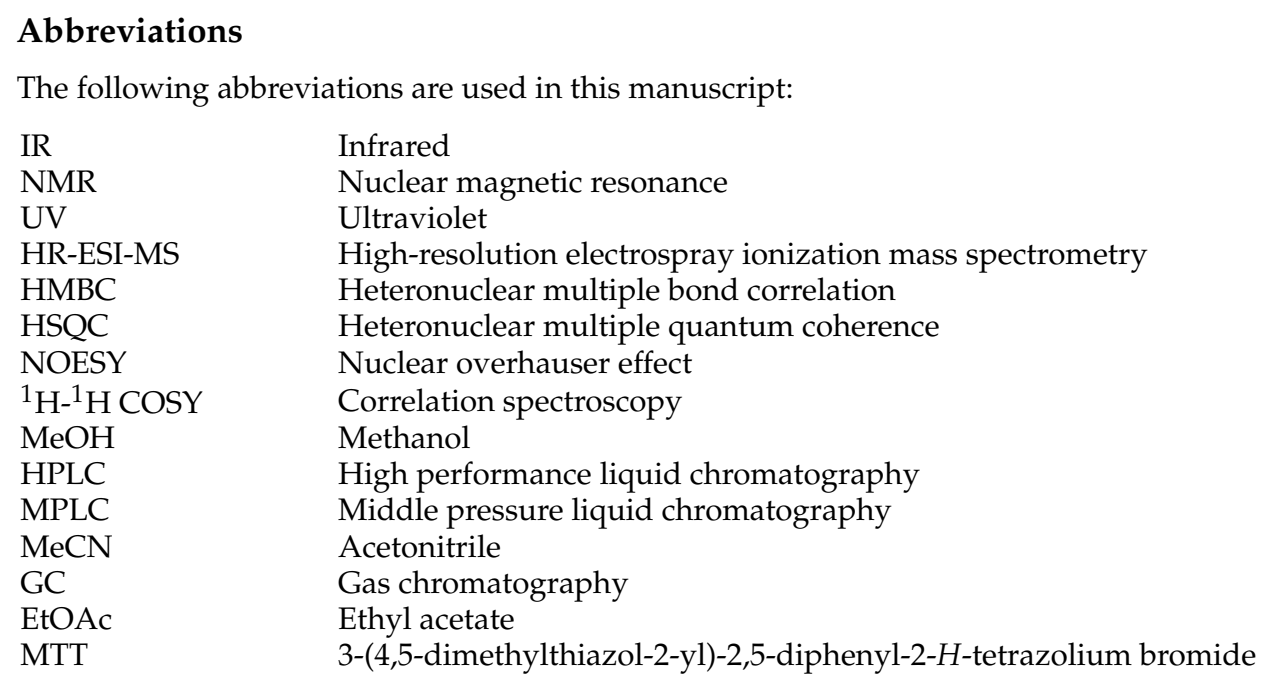

\section{References}

1. Wang, Y.L.; Dan, Y.; Yang, D.W.; Hu, Y.L.; Zhang, L.; Zhang, C.H.; Zhu, H.; Cui, Z.H.; Li, M.H.; Liu, Y.Z. The genus Anemarrhena Bunge: A review on ethnopharmacology, phytochemistry and pharmacology. J. Ethnopharmacol. 2014, 153, 42-46. [CrossRef] [PubMed]

2. Lim, S.M.; Jeong, J.J.; Kang, J.D.; Kim, K.A.; Choi, H.S.; Kim, D.H. Timosaponin AIII and its metabolite sarsasapogenin ameliorate colitis in mice by inhibiting NF- $\mathrm{KB}$ and MAPK activation and restoring Th17/Treg cell balance. Int. Immunopharm. 2015, 25, 493-503. [CrossRef] [PubMed]

3. Lu, W.Q.; Qiu, Y.; Li, T.J.; Tao, X.; Sun, L.N.; Chen, W.S. Antiplatelet and antithrombotic activities of timosaponin B-II, an extract of Anemarrhena asphodeloides. Clin. Exp. Pharmacol. Physiol. 2011, 38, 380-384. [CrossRef] [PubMed]

4. Zhang, J.Y.; Meng, Z.Y.; Ma, D.H.; Xu, S.X.; Kodama, H. Effect of six steroidal saponins isolated from anemarrhenae rhizoma on platelet aggregation and hemolysis in human blood. Clin. Chim. Acta 1999, 289, 78-88. [CrossRef] 
5. Kang, Y.J.; Chung, H.J.; Nam, J.W.; Park, H.J.; Seo, E.K.; Kim, Y.S.; Lee, D.; Lee, S.K. Cytotoxic and Antineoplastic Activity of Timosaponin A III for Human Colon Cancer Cells. J. Nat. Prod. 2011, 74, 701-706. [CrossRef] [PubMed]

6. Ni, Y.; Wang, Y.; Liu, M.; Chen, H.M.; Gong, X.G. Mitochondrial ROS burst as an early sign in sarsasapogenin induced apoptosis in HepG2 cells. Cell. Biol. Int. 2008, 32, 337-343. [CrossRef] [PubMed]

7. Dong, J.X.; Han, G.Y. Studies on the active constituents of Anemarrhena asphodeloides Bge. Acta Pharm. Sin. 1996, 27, 26-32.

8. Meng, Z.Y.; Zhou, X.M.; Xu, S.X. A New Steroidal Saponin from Anemarrhena asphodeloides Bge. J. Shenyang Pharm. Univ. 1998, 15, 254-256.

9. Meng, Z.Y.; Xu, S.X.; Meng, L.H. Timosaponin E1 and E2. Acta Pharm. Sin. 1998, 33, 693-696.

10. Qin, L.P.; Han, T.; Cao, D.P.; Zhang, Q.Y.; Nian, H.; Rahman, K.; Zheng, H.C. Antiosteoporotic chemical constituents from Er-Xian Decoction, a traditional Chinese herbal formula. J. Ethnopharmacol. 2008, 118, 271-279. [CrossRef] [PubMed]

11. Agrawal, P.K. Assigning stereo diversity of the 27-Me group of furostane-type steroidal saponins via NMR chemical shifts. Steroids 2005, 70, 715-724. [CrossRef] [PubMed]

12. Kostova, I.; Dinchev, D. Saponins in Tribulusterrestris-chemistry and bioactivity. Phytochemistry 2005, 4, 111-137. [CrossRef]

13. Ma, B.P.; Dong, J.X.; Yan, X.Z. Studies on the furostanol saponins from Anemarrhena asphodeloides Bunge. Acta Pharm. Sin. 1996, 31, 271-277.

14. Cheng, S.B.; Zhang, Y.F.; Wang, Y.; Wang, Y. Steroidal saponins from Allii Macrostemonis Bulbus. Chin. Tradit. Herb. Drugs 2013, 44, 1078-1081.

15. Yukiko, M.; Nana, A.; Chisato, H.; Fumito, T.; Yoshihiro, M. Steroidal glycosides from the bulbs of Besseraelegans and their cytotoxic activities. Phytochemistry 2013, 96, 244-256.

16. Setsuo, S.; Satoshi, N.; Koki, I. New Steroidal Saponins from the Rhizome of Anemarrhena asphodeloides Bunge (Liliaceae). Chem. Pharm. Bull. 1994, 42, 2342-2345.

17. Nagumo, S.; Kishi, S.; Inoue, T.; Nagai, M. Saponins of Anemarrhenae Rhizoma. Yakugaku Zasshi 1991, 111, 306-310. [PubMed]

18. Agrawal, P.K. $25 R / 25 S$ stereochemistry of spirostane-type steroidal sapogenins and steroidal saponins via chemical shift of geminal protons of ring-F. Magn. Reson. Chem. 2003, 41, 965-968. [CrossRef]

19. Yang, B.Y.; Guo, R.; Li, T.; Wu, J.J.; Zhang, J.; Liu, Y.; Wang, Q.H.; Kuang, H.X. New anti-inflammatory withanolides from the leaves of Datura metel L. Steroids 2014, 87, 26-34. [CrossRef] [PubMed]

20. Hussain, R.F.; Nouri, A.M.E.; Oliver, R.T.D. A new approach for measurement of cytotoxicity using colorimetric assay. J. Immunol. Methods 1993, 160, 89-96. [CrossRef]

21. Guo, C.R.; Li, L.; Yang, X.L.; Meng, Z.Q.; Li, F.; Zhang, C.F.; Yang, Z.L. Protective effects of timosaponin B II on high glucose-induced apoptosis in human umbilical vein endothelial cells. Environ. Toxicol. Pharmacol. 2014, 37, 37-44. [CrossRef] [PubMed]

Sample Availability: Not available.

(C) 2016 by the authors; licensee MDPI, Basel, Switzerland. This article is an open access article distributed under the terms and conditions of the Creative Commons Attribution (CC-BY) license (http://creativecommons.org/licenses/by/4.0/). 